\title{
Análise comparativa das curvas de mortes por covid-19 em catorze distritos do Município de São Paulo (Brasil)
}

\section{Comparative analysis of covid-19 deaths charts in fourteen districts in the city of São Paulo (Brazil)}

\author{
Sheila Regina Sarra ${ }^{1 *}$, Roberta Consentino Kronka Mülfarth ${ }^{1}$
}

\begin{abstract}
RESUMO
Com o objetivo de investigar a evolução do surto da doença do novo Coronavírus nas diversas regiões do Município de São Paulo, foram estudados os gráficos de mortes de catorze distritos no período de 44 semanas que sucederam à primeira morte. Os padrões dos gráficos obtidos foram comparados com dados sorológicos, demográficos, socioeconômicos, habitacionais, mobilidade, meios de transporte utilizados e disponibilidade de leitos hospitalares. Nos distritos mais afetados pela epidemia, as curvas de mortes tiveram evolução inicial mais acelerada e picos mais altos. Diversos fatores podem explicar os maiores impactos da epidemia nesses distritos, em especial o baixo nível socioeconômico, a precariedade das habitações, a carência de infraestrutura, a falta de serviços públicos essenciais, e a falta de leitos hospitalares. Conclui-se que os impactos da epidemia do novo Coronavírus estão muito interligados às questões socioeconômicas, habitacionais e de infraestrutura urbana e hospitalar.
\end{abstract}

Palavras-chave: Novo coronavírus; Epidemia; Vulnerabilidade.

\section{ABSTRACT}

In order to investigate the new Coronavirus disease 2019 outbreak evolution in different regions in the city of São Paulo, we studied the deaths charts of fourteen districts during the 44 weeks that succeeded the first death. We compared the pattern of the graphics with serological, demographic, socioeconomic and habitational data, mobility, means of transport used and availability of hospital beds. In the districts hardest hit by the epidemic, the death charts have shown fast initial evolution and highest peaks. Several factors may be invoked to explain the most severe impacts in these districts, specially, socioeconomic problems, lack of infrastructure, lack of basic public services and lack of hospital beds. We concluded that the impacts of the new Coronavirus epidemics are associated with socioeconomic, habitational, urban infrastructure and hospital network issues.

Keywords: New coronavirus; EpidemicVulnerability.

${ }^{1}$ Universidade de São Paulo, Faculdade de Arquitetura e Urbanismo, Departamento de Tecnologia.

*E-mail: sheila_sarra@hotmail.com 


\section{INTRODUÇÃO}

A epidemia provocada pelo coronavírus 2 (SARS-CoV-2) evoluiu de forma rápida nos ambientes urbanos, trazendo uma série de restrições aos seus moradores. Segundo Alirol et al (2011), diversos fatores podem ter contribuído para maior transmissão no ambiente urbano: 1) maior densidade populacional e proximidade das pessoas, 2) presença de desigualdades sociais e segregação de parte da população, 3) transporte público lotado, 4) espaços públicos congestionados e aglomerações nas áreas de lazer, 5) acesso diferenciado ao sistema de saúde, 6) alto fluxo de imigrantes, 7) poluição ambiental, 8) adaptação dos agentes patogênicos ao ambiente urbano.

Segundo Pan et al. (2020), a alta proporção de casos assintomáticos foi um dos fatores que aumentou a transmissão da doença em suas fases iniciais, justificando a adoção de medidas de distanciamento social. Segundo Nussbaumer-Streit et al. (2020), a associação de medidas de quarentena (isolamento de pessoas expostas a casos suspeitos ou confirmados) com outras medidas de saúde pública (distanciamento social, fechamento de escolas, restrição de viagens), possibilitou redução na transmissão da doença. Ferguson et al. (2020) apontam que o combate à epidemia de Covid-19 é feito pelo isolamento dos doentes e pelo distanciamento social. Quando o distanciamento social atinge apenas a população com maior risco de complicações, o objetivo é reduzir o número de hospitalizações (mitigação). Quando o distanciamento social inclui toda população, o objetivo é reverter o crescimento da epidemia (supressão). Durante a pandemia, as duas medidas são importantes, porém, é preciso lembrar que as populações têm comportamentos próprios e respondem de forma diferente às medidas recomendadas.

Estudos de Jiang e Luo (2020) mostraram que a transmissão respiratória do coronavírus 2 (SARS-CoV-2) está bastante relacionada com a mobilidade urbana e o uso do transporte público. Neste sentido, as medidas de "lockdown" foram importantes parar reduzir o ritmo de transmissão, embora tenham trazido severos impactos econômicos. Para Bonaccorsi et al. (2020), as restrições da mobilidade urbana se mostraram bastante eficientes em reduzir a taxa de transmissão do novo coronavírus, especialmente nas cidades com condições de fiscalização. Segundo Kraemer et al. (2020), a implementação de medidas de controle e restrição do deslocamento da população contém a expansão da doença, especialmente nas fases iniciais da epidemia, quando ela ainda está confinada a algumas regiões. 
Foram também reconhecidas várias condições de vulnerabilidade, capazes de aumentar a letalidade da doença. Segundo Jardim et al. (2020), a pandemia levou a uma maior letalidade em regiões com baixos níveis de educação e renda, maior número de viagens ao trabalho e com falhas no abastecimento de água. Estudo de Verity et al. (2020), mostrou que as taxas de letalidade por Covid-19 aumentam significativamente com a idade. Os valores dependem da qualidade do atendimento médico e da disponibilidade de leitos hospitalares.

\section{METODOLOGIA}

Foram estudados catorze distritos do Município de São Paulo (MSP). A localização dos distritos no MSP e as respectivas regiões podem ser vistas na Figura 1.

Figura 1 - Mapa do MSP com os distritos analisados e as respectivas regiões

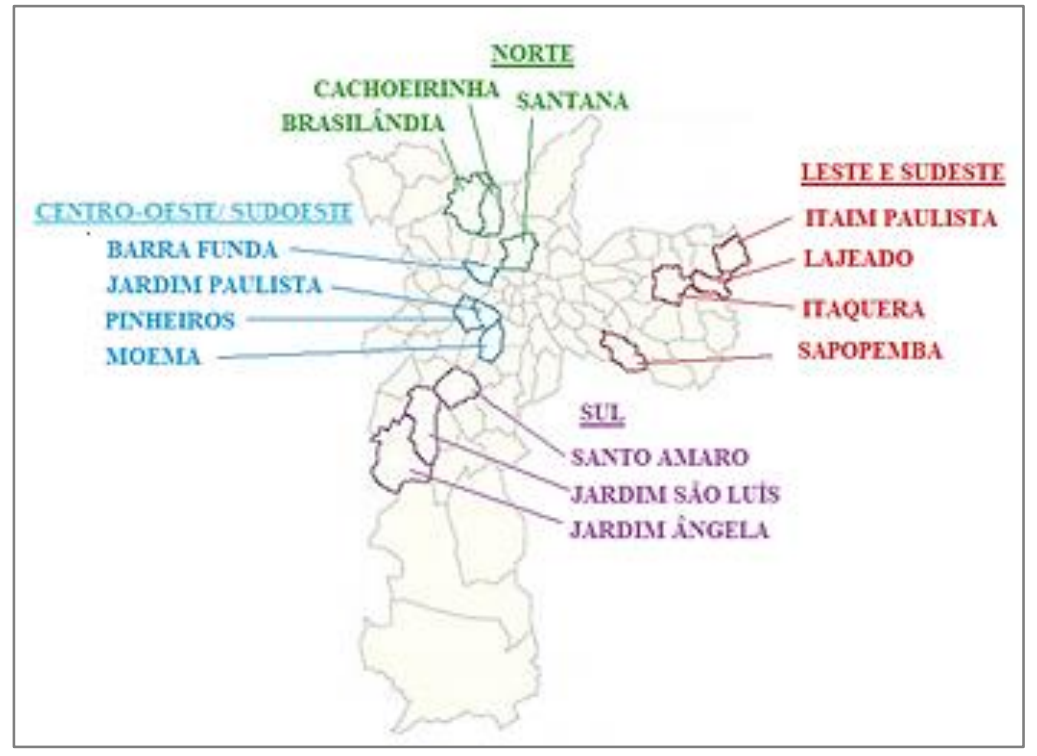

Fonte: elaborado pelo autor a partir do mapa do Município de São Paulo (2020

O estudo compara as curvas de óbitos por Covid-19 com os dados sorológicos, demográficos, socioeconômicos, habitacionais, mobilidade, meios de transporte e disponibilidade de leitos hospitalares em cada distrito.

Utilizou-se o Sistema DATASUS (Departamento de Informática do Sistema Único de Saúde) para correlacionar os óbitos com a área geográfica de residência e os dados demográficos. Nos dados demográficos considerou-se o Índice de Envelhecimento como a relação entre o número de pessoas com 60 anos ou mais para cada 100 pessoas com menos de 15 anos de idade. O Índice de mobilidade é o número de viagens diárias 
por habitante. O tempo médio de deslocamento ao trabalho inclui as viagens de ida e volta. O coeficiente de leitos hospitalares gerais é medido por 1.000 habitantes. Gonçalves (1972) considera aceitável o valor acima de 4 leitos por 1.000 habitantes.

O período estudado vai da data do primeiro óbito no MSP (ocorrido na $11^{\mathrm{a}}$ semana epidemiológica) até a $44^{\mathrm{a}}$ semana epidemiológica. O Quadro 1 resume os dados utilizados e as respectivas fontes.

Quadro 1 - Dados utilizados no estudo e respectivas fontes

\begin{tabular}{|l|l|l|}
\hline \multicolumn{2}{|c|}{ DADOS (11 ${ }^{\text {a }}$ A $44^{a}$ SEMANA EPIDEMIOLÓGICA) } & \multicolumn{1}{c|}{ FONTE } \\
\hline \multirow{2}{*}{ Epidemiológicos } & $\begin{array}{l}\text { Óbitos por Covid-19 por semana } \\
\text { epidemiológica e por distrito }\end{array}$ & DATASUS \\
\cline { 2 - 3 } & $\begin{array}{l}\text { Óbitos por Covid-19 por 1000 habitantes do } \\
\text { distrito e distribuição por faixa etária }\end{array}$ & DATASUS \\
\hline \multirow{2}{*}{ Soroepidemiológicos } & $\begin{array}{l}\text { Estudo SoroEpi de soroprevalência de } \\
\text { COVID-19 }\end{array}$ & SoroEpi MSP \\
\hline \multirow{2}{*}{ Demográficos } & População (2020) & Prefeitura do MSP \\
\cline { 2 - 3 } & Índice de Envelhecimento (2019) & Prefeitura do MSP \\
\hline Socioeconômicos & Renda mensal média (2010) & Prefeitura do MSP \\
\hline \multirow{2}{*}{ Saneamento } & $\begin{array}{l}\text { Domicílios não ligados à rede de esgoto } \\
\text { (2010) }\end{array}$ & Prefeitura do MSP \\
\hline \multirow{3}{*}{ Moradia } & Densidade por dormitório (2010) & Prefeitura do MSP \\
\cline { 2 - 3 } & Número de favelas (2017) & Prefeitura do MSP \\
\cline { 2 - 3 } & Número de domicílios em favelas (2017) & Prefeitura do MSP \\
\hline \multirow{2}{*}{$\begin{array}{l}\text { Mobilidade e meios } \\
\text { de transporte }\end{array}$} & Índice de mobilidade da população (2017) & Prefeitura do MSP \\
\cline { 2 - 3 } & Mobilidade por meio de transporte (2017) & Prefeitura do MSP \\
\cline { 2 - 3 } & $\begin{array}{l}\text { Tempo médio de deslocamento ao trabalho } \\
\text { (2017) }\end{array}$ & Prefeitura do MSP \\
\hline $\begin{array}{l}\text { Coeficiente de leitos } \\
\text { hospitalares }\end{array}$ & Leitos/1.000 habitantes (2019) & Prefeitura do MSP \\
\hline
\end{tabular}

\section{RESULTADOS DO ESTUDO}

Iniciaremos com os distritos das regiões Centro-oeste e Sudoeste: Barra Funda, Jardim Paulista, Moema e Pinheiros. Os gráficos de óbitos por Covid-19 por distrito estão na Figura 2. Nota-se que as curvas permaneceram com médias baixas $(0,82 ; 3,74 ; 3,59$ e 2,74 respectivamente) e desvios-padrão pequenos $(1,09 ; 2,79 ; 2,68$ e 2,08 respectivamente). 
Figura 2 - Curvas de óbitos nos distritos de Barra Funda, Jardim Paulista, Moema e Pinheiros

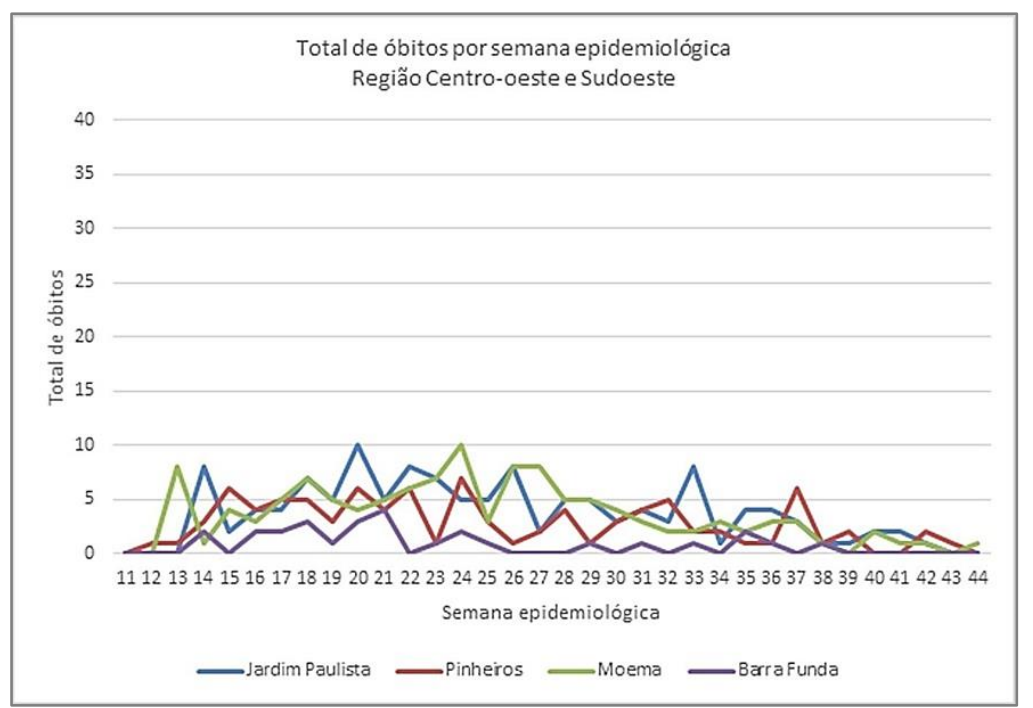

Fonte: Autor a partir de dados do DATASUS (2020)

Os estudos soroepidemiológicos realizados entre 15 e 24 de junho de 2020 mostraram soroprevalência para Covid-19 de 6,5\% nos quatro distritos. No estudo repetido entre 01 e 10 de outubro, a soroprevalência passou para $21,6 \%$ nos quatro distritos. O Quadro 2 mostra, para cada distrito, os dados de epidemiologia, demografia, leitos hospitalares, condições dos domicílios, mobilidade e tempo de deslocamento ao trabalho.

Quadro 2 - Dados dos distritos de Barra Funda, Jardim Paulista, Moema e Pinheiros

\begin{tabular}{|c|c|c|c|c|}
\hline Epidemiologia & $\begin{array}{c}\text { Óbitos até a 44 } \\
\text { Semana } \\
\text { Epidemiológica }\end{array}$ & \multirow{2}{*}{$\begin{array}{c}\text { Óbitos por } 1.000 \\
\text { habitantes do Distrito }\end{array}$} & \multicolumn{2}{|c|}{ Óbitos por faixa etária } \\
\cline { 4 - 5 } & 28 & 1,9 & 65 anos & $>65$ anos \\
\hline Barra Funda & 127 & 1,4 & $10(8 \%)$ & $117(92 \%)$ \\
\hline J. Paulista & 122 & 1,4 & $12(10 \%)$ & $110(90 \%)$ \\
\hline Moema & 93 & 1,4 & $9(10 \%)$ & $84(90 \%)$ \\
\hline Pinheiros & População & $\begin{array}{c}\text { Índice de } \\
\text { Envelhecimento }\end{array}$ & $\begin{array}{c}\text { Renda } \\
\text { Ménsal }\end{array}$ & $\begin{array}{c}\text { Coeficiente } \\
\text { de leitos } \\
\text { gerais }\end{array}$ \\
\hline $\begin{array}{c}\text { Demografia e } \\
\text { hospitais }\end{array}$ & 14.383 & 117,4 & $3.927,67$ & $\begin{array}{c}0,96 / 1.000 \\
\text { habs }\end{array}$ \\
\hline Barra Funda & 88.692 & 205,2 & $6.647,48$ & $\begin{array}{c}10,84 / 1.000 \\
\text { habs }\end{array}$ \\
\hline J. Paulista & 83.368 & 187,9 & $7.384,73$ & $\begin{array}{c}12,44 / 1.000 \\
\text { habs }\end{array}$ \\
\hline Moema & 65.364 & 194,4 & $5.466,61$ & $\begin{array}{c}10,84 / 1.000 \\
\text { habs }\end{array}$ \\
\hline Pinheiros & & & &
\end{tabular}




\begin{tabular}{|c|c|c|c|c|c|c|c|}
\hline \multirow[t]{2}{*}{ Domicílios } & \multirow{2}{*}{$\begin{array}{c}\text { Domicílios não } \\
\text { ligados à rede } \\
\text { de esgoto }\end{array}$} & \multicolumn{3}{|c|}{$\begin{array}{c}\text { Densidade de pessoas por } \\
\text { dormitório }\end{array}$} & \multirow{2}{*}{\multicolumn{2}{|c|}{$\begin{array}{l}\text { Número de } \\
\text { favelas }\end{array}$}} & \multirow{2}{*}{$\begin{array}{l}\text { Domicílios } \\
\text { em favelas }\end{array}$} \\
\hline & & Até 1 & 1 a 3 & $>3$ & & & \\
\hline Barra Funda & $254(4 \%)$ & $56,5 \%$ & $41 \%$ & $12,5 \%$ & 3 & & 70 \\
\hline J. Paulista & $37(0,09 \%)$ & $69 \%$ & $30,5 \%$ & $0,5 \%$ & 0 & & 0 \\
\hline Moema & $0(0 \%)$ & $66 \%$ & $33,8 \%$ & $0,2 \%$ & 0 & & 0 \\
\hline Pinheiros & $19(0,07 \%)$ & $63,1 \%$ & $36 \%$ & $0,9 \%$ & 2 & & 22 \\
\hline $\begin{array}{l}\text { Mobilidade e } \\
\text { transporte }\end{array}$ & \multicolumn{5}{|c|}{ Índice de Mobilidade } & \multicolumn{2}{|c|}{$\begin{array}{c}\text { Tempo médio de } \\
\text { deslocamento ao } \\
\text { trabalho }\end{array}$} \\
\hline Barra Funda & $\begin{array}{c}\text { Motorizado Indi } \\
0,74 \\
\text { Motorizado Co } \\
0,60 \\
\end{array}$ & $\begin{array}{l}\text { idual - } \\
\text { etivo - }\end{array}$ & $\begin{array}{l}\text { A pé } \\
0,61\end{array}$ & $\begin{array}{c}\text { Bicicleta } \\
0,03\end{array}$ & $\begin{array}{l}\text { Total } \\
2,04\end{array}$ & \multicolumn{2}{|c|}{ Menos de 1 hora } \\
\hline J. Paulista & $\begin{array}{r}\text { Motorizado Ind } \\
-1,04 \\
\text { Motorizado Col } \\
0,61 \\
\end{array}$ & vidual & $\begin{array}{l}\text { A pé } \\
0,74\end{array}$ & $\begin{array}{c}\text { Bicicleta } \\
0,04\end{array}$ & $\begin{array}{l}\text { Total } \\
2,44\end{array}$ & \multicolumn{2}{|c|}{ Menos de 1 hora } \\
\hline Moema & $\begin{array}{r}\text { Motorizado Ind } \\
-1,43 \\
\text { Motorizado Co } \\
0,39 \\
\end{array}$ & vidual & $\begin{array}{l}\text { A pé } \\
0,69\end{array}$ & $\begin{array}{c}\text { Bicicleta } \\
0,06\end{array}$ & $\begin{array}{c}\text { Total } \\
2,56\end{array}$ & \multicolumn{2}{|c|}{ Menos de 1 hora } \\
\hline Pinheiros & $\begin{array}{c}\text { Motorizado Indi } \\
0,97 \\
\text { Motorizado Co } \\
0,65\end{array}$ & $\begin{array}{l}\text { idual - } \\
\text { etivo - }\end{array}$ & $\begin{array}{l}\text { A pé } \\
0,67\end{array}$ & $\begin{array}{c}\text { Bicicleta } \\
0,10\end{array}$ & $\begin{array}{l}\text { Total } \\
2,39\end{array}$ & \multicolumn{2}{|c|}{ Menos de 1 hora } \\
\hline
\end{tabular}

O conjunto de dados mostra que os distritos analisados tiveram curvas de mortes que se mantiveram em níveis baixos durante o período do estudo. Nesses distritos, as medidas de distanciamento social conseguiram inibir o crescimento da curva de óbitos. Com relação à mortalidade, apesar de terem um índice de envelhecimento elevado, os distritos de Jardim Paulista, Moema e Pinheiros tiveram baixo número de óbitos por 1000 habitantes. Como fatores para este resultado, pode-se mencionar a renda mensal média elevada, a baixa densidade de pessoas por dormitório, o maior uso de transporte individual, o número baixo ou inexistente de favelas, a menor porcentagem de domicílios não ligados à rede de esgoto e alto coeficiente de leitos hospitalares. $\mathrm{O}$ distrito da Barra Funda, apesar de ser um bairro com população mais jovem, teve maior porcentagem de óbitos por 1000 habitantes, notando-se que $21 \%$ dos óbitos ocorreram em pessoas com menos de 65 anos. Para isto, podem ter contribuído os seguintes fatores: menor renda mensal média, maior densidade de pessoas por dormitório, menor uso de transporte 
individual, maior número de favelas, maior porcentagem de domicílios não ligados à rede de esgoto e baixo coeficiente de leitos por 1.000 habitantes.

Em seguida são fornecidos os resultados dos distritos da região Sul: Santo Amaro, Jardim São Luís e Jardim Ângela. Os gráficos de óbitos por Covid-19 por distrito estão na Figura 3. A curva do distrito de Santo Amaro tem lenta ascensão seguida de um declínio gradual, mostrando o efeito das medidas de distanciamento social. A média $(4,0)$ e o desvio padrão $(3,68)$ são baixos, mas acima dos encontrados nos distritos da região Centro-oeste/Sudoeste. As curvas do Jardim São Luís e do Jardim Ângela mostram rápida e acentuada ascensão, com pico acentuado entre a $19^{\mathrm{a}}$ e a $20^{\mathrm{a}}$ semanas. Dificuldades para aderir ao distanciamento social podem ter impulsionado a transmissão da doença. As médias foram altas (11,97 e 11,82 respectivamente) e os desvios-padrão (8,90 e 10,14 respectivamente) elevados.

Figura 3 - Curvas de óbitos nos distritos de Santo Amaro, Jardim São Luís e Jardim Ângela

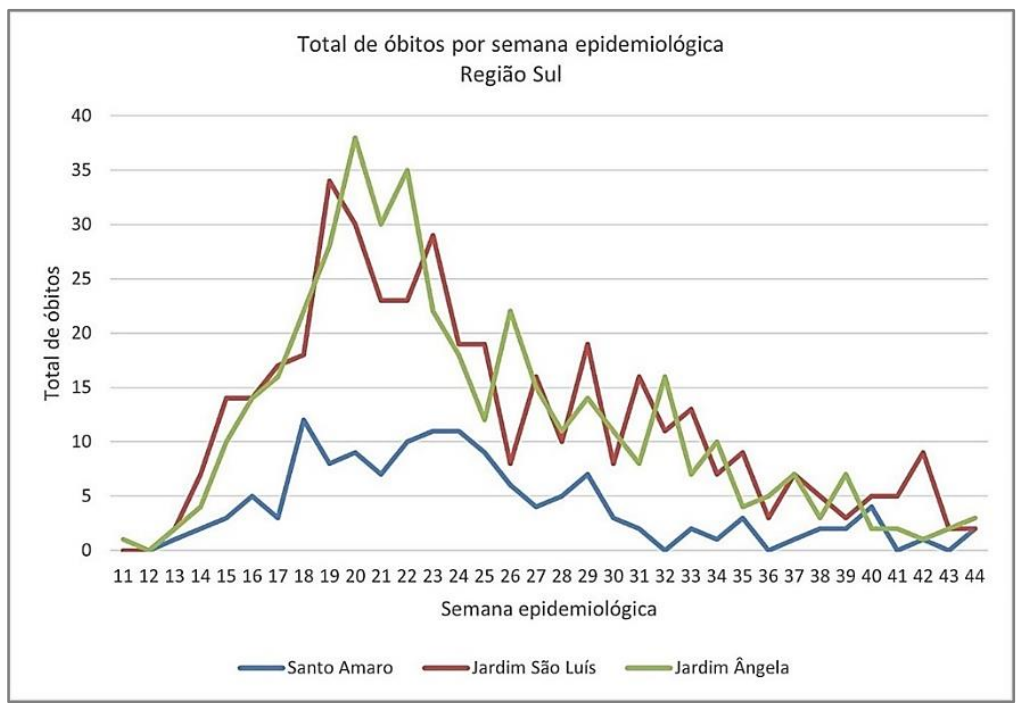

Fonte: Autor a partir de dados do DATASUS (2020)

Os estudos soroepidemiológicos realizados entre 15 e 24 de junho de 2020 mostraram soroprevalência para Covid-19 de 6,5\% em Santo Amaro e 16\% no Jardim São Luís e no Jardim Ângela. No estudo repetido entre 01 e 10 de outubro, a soroprevalência passou para 21,6\% em Santo Amaro e para 30,4\% no Jardim São Luís e no Jardim Ângela. O Quadro 3 mostra, para cada distrito, os dados de epidemiologia, demografia, leitos hospitalares, condições dos domicílios, mobilidade e tempo de deslocamento ao trabalho. 
Quadro 3 - Dados dos distritos de Santo Amaro, Jardim São Luís e Jardim Ângela

\begin{tabular}{|c|c|c|c|c|c|c|c|}
\hline \multirow{2}{*}{ Epidemiologia } & \multirow{2}{*}{$\begin{array}{l}\text { Óbitos até a } 44^{\mathrm{a}} \\
\text { Semana } \\
\text { Epidemiológica } \\
\end{array}$} & \multirow{2}{*}{\multicolumn{3}{|c|}{$\begin{array}{l}\text { Óbitos por } 1.000 \\
\text { habitantes do Distrito }\end{array}$}} & \multicolumn{3}{|c|}{ Óbitos por faixa etária } \\
\hline & & & & & \multicolumn{2}{|c|}{$<65$ anos } & $>65$ anos \\
\hline Santo Amaro & 139 & \multicolumn{3}{|c|}{1,9} & \multicolumn{2}{|c|}{$18(13$} & $121(87 \%)$ \\
\hline Jardim S. Luís & 407 & \multicolumn{3}{|c|}{1,5} & \multicolumn{2}{|c|}{$132(32 \%)$} & $275(68 \%)$ \\
\hline Jardim Ângela & 402 & \multicolumn{3}{|c|}{1,4} & $157(3$ & & $245(61 \%)$ \\
\hline $\begin{array}{l}\text { Demografia e } \\
\text { hospitais }\end{array}$ & População & \multicolumn{3}{|c|}{ 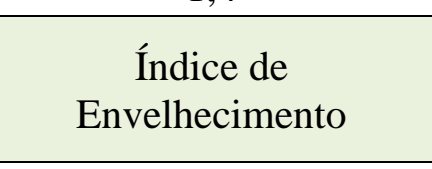 } & \multicolumn{2}{|c|}{$\begin{array}{l}\text { Renda } \\
\text { Mensal } \\
\text { Média }\end{array}$} & $\begin{array}{c}\text { Coeficiente } \\
\text { de leitos } \\
\text { gerais } \\
\end{array}$ \\
\hline Santo Amaro & 71.560 & \multicolumn{3}{|c|}{168,5} & \multicolumn{2}{|c|}{$4.834,04$} & $\begin{array}{c}2,94 / 1.000 \\
\text { habs }\end{array}$ \\
\hline Jardim S. Luís & 267.871 & \multicolumn{3}{|c|}{55,3} & \multicolumn{2}{|c|}{$1.110,48$} & $\begin{array}{c}0,66 / 1.000 \\
\text { habs }\end{array}$ \\
\hline Jardim Ângela & 295.434 & \multicolumn{3}{|c|}{39,1} & \multicolumn{2}{|c|}{887,97} & $\begin{array}{c}0,66 / 1.000 \\
\text { habs }\end{array}$ \\
\hline \multirow{2}{*}{ Domicílios } & \multirow{2}{*}{$\begin{array}{l}\text { Domicílios não } \\
\text { ligados à rede } \\
\text { de esgoto }\end{array}$} & \multicolumn{3}{|c|}{$\begin{array}{c}\text { Densidade de pessoas } \\
\text { por dormitório }\end{array}$} & \multirow{2}{*}{\multicolumn{2}{|c|}{$\begin{array}{l}\text { Número de } \\
\text { favelas }\end{array}$}} & \multirow{2}{*}{$\begin{array}{l}\text { Domicílios } \\
\text { em favelas }\end{array}$} \\
\hline & & $\begin{array}{c}\text { Até } \\
1\end{array}$ & 1 a 3 & $>3$ & & & \\
\hline Santo Amaro & $396(1,6 \%)$ & $53 \%$ & $46 \%$ & $1 \%$ & \multicolumn{2}{|c|}{1} & 44 \\
\hline Jardim S. Luís & $7.716(9 \%)$ & $25 \%$ & $64 \%$ & $11 \%$ & \multicolumn{2}{|c|}{75} & 19.903 \\
\hline Jardim Ângela & $18.432(21 \%)$ & $20 \%$ & $66 \%$ & $14 \%$ & \multicolumn{2}{|c|}{89} & 22.447 \\
\hline $\begin{array}{l}\text { Mobilidade e } \\
\text { transporte }\end{array}$ & \multicolumn{5}{|c|}{ Índice de Mobilidade } & \multicolumn{2}{|c|}{$\begin{array}{c}\text { Tempo médio de } \\
\text { deslocamento ao } \\
\text { trabalho }\end{array}$} \\
\hline Santo Amaro & \multicolumn{2}{|c|}{$\begin{array}{c}\text { Motorizado Individual } \\
-1,23 \\
\text { Motorizado Coletivo - } \\
0,51 \\
\end{array}$} & $\begin{array}{l}\text { A pé } \\
0,65\end{array}$ & $\begin{array}{l}\text { Bicicleta } \\
0,02\end{array}$ & $\begin{array}{l}\text { Total } \\
2,41\end{array}$ & \multicolumn{2}{|c|}{$1: 00$ a $1: 15 \mathrm{~h}$} \\
\hline Jardim S. Luís & \multicolumn{2}{|c|}{$\begin{array}{c}\text { Motorizado Individual } \\
-0,37 \\
\text { Motorizado Coletivo - } \\
0,95 \\
\end{array}$} & $\begin{array}{l}\text { A pé } \\
0,72\end{array}$ & $\begin{array}{c}\text { Bicicleta } \\
0,01\end{array}$ & $\begin{array}{l}\text { Total } \\
2,04\end{array}$ & \multicolumn{2}{|c|}{$1: 30$ a $2: 00 \mathrm{~h}$} \\
\hline Jardim Ângela & \multicolumn{2}{|c|}{$\begin{array}{c}\text { Motorizado Individual } \\
-0,3 \\
\text { Motorizado Coletivo - } \\
1,02\end{array}$} & $\begin{array}{l}\text { A pé } \\
0,654\end{array}$ & $\begin{array}{c}\text { Bicicleta } \\
0\end{array}$ & $\begin{array}{c}\text { Total } \\
1,85\end{array}$ & \multicolumn{2}{|c|}{$2: 00$ a $2: 30 \mathrm{~h}$} \\
\hline
\end{tabular}

O conjunto de dados mostra que os distritos de Jardim São Luís e Jardim Ângela tiveram curvas de óbitos caracterizadas por rápido e acentuado crescimento inicial, refletindo as dificuldades de implementação das medidas de distanciamento social. Diversos fatores socioeconômicos podem ter contribuído, incluindo a precariedade das 
habitações com elevado número de domicílios não-ligados à rede de esgoto, alta densidade de pessoas por dormitório, e elevado número de favelas. $\mathrm{O}$ uso de transporte coletivo com elevado tempo de deslocamento ao trabalho também pode ter favorecido a disseminação da doença. No distrito de Santo Amaro, a curva de mortes foi mais achatada, refletindo a implementação das medidas de distanciamento social. Porém, em consequência da maior faixa etária da população, a mortalidade atingiu 1,9 mortos/1.000 habitantes, predominando acima de 65 anos (87\%). Os coeficientes de leitos hospitalares do Jardim São Luís e do Jardim Ângela são muito reduzidos, indicando importante limitação na capacidade de atendimento médico. No distrito de Santo Amaro, a proporção também é baixa, porém num nível superior.

Em seguida, estão os resultados dos distritos da região Norte: Cachoeirinha, Brasilândia e Santana. Os gráficos de óbitos por Covid-19 por distrito estão na Figura 4. Nos distritos de Cachoeirinha e Brasilândia, a alta transmissão inicial da doença levou a uma curva de evolução inicial acelerada, atingindo os picos máximos na $17^{\mathrm{a}}$ semana e na $20^{\mathrm{a}}$ semana, respectivamente. São curvas com picos proeminentes, médias altas $(8,15$ e 13,85 respectivamente) e desvios-padrão altos (6,77 e 9,40 respectivamente). Em Santana, a curva foi mais achatada, refletindo a maior adesão às medidas de distanciamento social. A média $(7,15)$ e o desvio padrão $(4,86)$ são mais baixos

Figura 4 - Curvas de óbitos nos distritos de Cachoeirinha, Brasilândia e Santana

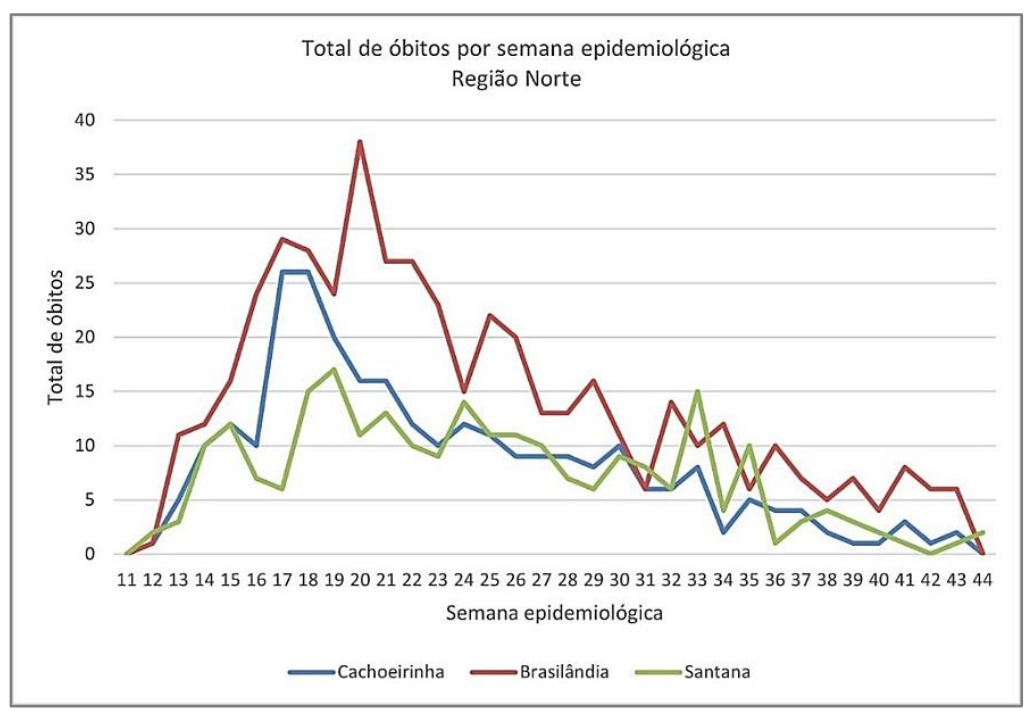

Fonte: Autor a partir de dados do DATASUS (2020)

Os estudos soroepidemiológicos realizados entre 15 e 24 de junho de 2020 mostraram soroprevalência para Covid-19 de 6,5\% em Santana e de 16\% nos distritos de 
Cachoeirinha e Brasilândia. No estudo repetido entre 01 e 10 de outubro, a soroprevalência passou para 21,6\% em Santana e para 30,4\% nos distritos de Cachoeirinha e Brasilândia. O Quadro 4 mostra os dados de epidemiologia, demografia, leitos hospitalares, domicílios, mobilidade e tempo de deslocamento ao trabalho.

Quadro 4 - Dados dos distritos de Cachoeirinha, Brasilândia e Santana

\begin{tabular}{|c|c|c|c|c|c|c|c|}
\hline \multirow{2}{*}{ Epidemiologia } & \multirow{2}{*}{$\begin{array}{l}\text { Total de óbitos } \\
\text { até a } 44^{\mathrm{a}} \\
\text { Semana } \\
\text { Epidemiológica }\end{array}$} & \multirow{2}{*}{\multicolumn{3}{|c|}{$\begin{array}{c}\text { Óbitos por } 1.000 \\
\text { habitantes do Distrito }\end{array}$}} & \multicolumn{3}{|c|}{ Óbitos por faixa etária } \\
\hline & & & & & \multicolumn{2}{|c|}{$<65$ anos } & $>65$ anos \\
\hline Cachoeirinha & 277 & \multicolumn{3}{|c|}{1,9} & \multicolumn{2}{|c|}{$92(33 \%)$} & $185(67 \%)$ \\
\hline Brasilândia & 471 & \multicolumn{3}{|c|}{1,8} & \multicolumn{2}{|c|}{$164(35 \%)$} & $307(65 \%)$ \\
\hline Santana & 243 & \multicolumn{3}{|c|}{2,0} & \multicolumn{2}{|c|}{$35(14 \%)$} & $208(86 \%)$ \\
\hline $\begin{array}{l}\text { Demografia e } \\
\text { hospitais }\end{array}$ & População & \multicolumn{3}{|c|}{$\begin{array}{c}\text { Índice de } \\
\text { Envelhecimento }\end{array}$} & \multicolumn{2}{|c|}{$\begin{array}{l}\text { Renda } \\
\text { Mensal } \\
\text { Média } \\
\end{array}$} & $\begin{array}{c}\text { Coeficiente } \\
\text { de leitos } \\
\text { gerais } \\
\end{array}$ \\
\hline Cachoeirinha & 143.523 & \multicolumn{3}{|c|}{58,6} & \multicolumn{2}{|c|}{$1.276,79$} & $\begin{array}{c}0,95 / 1.000 \\
\text { habs }\end{array}$ \\
\hline Brasilândia & 264.918 & \multicolumn{3}{|c|}{47,7} & \multicolumn{2}{|c|}{982,56} & $\begin{array}{c}0,41 / 1.000 \\
\text { habs }\end{array}$ \\
\hline Santana & 118.797 & \multicolumn{3}{|c|}{164,0} & \multicolumn{2}{|c|}{$3.159,52$} & $\begin{array}{c}3,70 / 1.000 \\
\text { habs }\end{array}$ \\
\hline \multirow{2}{*}{ Domicílios } & \multirow{2}{*}{$\begin{array}{l}\text { Domicílios não } \\
\text { ligados à rede } \\
\text { de esgoto }\end{array}$} & \multicolumn{3}{|c|}{$\begin{array}{l}\text { Densidade de pessoas } \\
\text { por dormitório }\end{array}$} & \multirow{2}{*}{\multicolumn{2}{|c|}{$\begin{array}{l}\text { Número de } \\
\text { favelas }\end{array}$}} & \multirow{2}{*}{$\begin{array}{l}\text { Domicílios } \\
\text { em favelas }\end{array}$} \\
\hline & & $\begin{array}{c}\text { Até } \\
1\end{array}$ & 1 a 3 & $>3$ & & & \\
\hline Cachoeirinha & $3.811(9 \%)$ & $25 \%$ & $65 \%$ & $10 \%$ & \multicolumn{2}{|c|}{35} & 9.605 \\
\hline Brasilândia & $9.548(12 \%)$ & $21 \%$ & $65 \%$ & $14 \%$ & \multicolumn{2}{|c|}{94} & 22.793 \\
\hline Santana & $413(1 \%)$ & $49 \%$ & $49 \%$ & $2 \%$ & \multicolumn{2}{|c|}{4} & 96 \\
\hline $\begin{array}{l}\text { Mobilidade e } \\
\text { transporte }\end{array}$ & \multicolumn{5}{|c|}{ Índice de Mobilidade } & \multicolumn{2}{|c|}{$\begin{array}{l}\text { Tempo médio de } \\
\text { deslocamento ao } \\
\text { trabalho }\end{array}$} \\
\hline Cachoeirinha & \multicolumn{2}{|c|}{$\begin{array}{c}\text { Motorizado Individual } \\
-0,58 \\
\text { Motorizado Coletivo - } \\
0,91 \\
\end{array}$} & $\begin{array}{l}\text { A pé } \\
0,74\end{array}$ & $\begin{array}{l}\text { Bicicleta } \\
0,02\end{array}$ & $\begin{array}{l}\text { Total } \\
2,26\end{array}$ & \multicolumn{2}{|c|}{$1: 15$ a $1: 30 \mathrm{~h}$} \\
\hline Brasilândia & \multicolumn{2}{|c|}{$\begin{array}{c}\text { Motorizado Individual } \\
-0,4 \\
\text { Motorizado Coletivo - } \\
0,9\end{array}$} & $\begin{array}{l}\text { A pé } \\
0,57\end{array}$ & $\begin{array}{c}\text { Bicicleta } \\
0\end{array}$ & $\begin{array}{c}\text { Total } \\
1,87\end{array}$ & \multicolumn{2}{|c|}{$1: 30$ a $2: 00 \mathrm{~h}$} \\
\hline Santana & \multicolumn{2}{|c|}{$\begin{array}{c}\text { Motorizado Individual } \\
-0,9 \\
\text { Motorizado Coletivo - } \\
0,8\end{array}$} & $\begin{array}{l}\text { A pé } \\
0,68\end{array}$ & $\begin{array}{c}\text { Bicicleta } \\
0\end{array}$ & $\begin{array}{l}\text { Total } \\
2,38\end{array}$ & \multicolumn{2}{|c|}{$1: 00$ a $1: 15 \mathrm{~h}$} \\
\hline
\end{tabular}


O conjunto de dados mostra que as curvas de óbitos com evolução inicial acelerada e picos elevados que ocorreram nos distritos de Cachoeirinha e Brasilândia, estão associadas à presença de populações de grande vulnerabilidade social e condições econômicas precárias. A população que vive em favelas é grande e a densidade de pessoas por dormitório é elevada. O transporte mais utilizado é o motorizado coletivo, sendo alto o tempo médio de deslocamento ao trabalho. Verificou-se, também, que esses distritos possuem coeficiente de leitos bastante baixos, dificultando o atendimento médico da população.

No distrito de Santana, a curva de óbitos foi mais achatada e não atingiu pico tão elevado. As condições socioeconômicas da população são melhores, a porcentagem de domicílios não ligados à rede de esgoto é pequena e o número de favelas é bem menor. A densidade de pessoas por dormitório é menor e o principal meio de transporte é o motorizado individual. Como o índice de envelhecimento da população é maior, justificase a maior mortalidade por mil habitantes em função da maior proporção de idosos.

Em seguida, são fornecidos os resultados dos distritos das regiões Leste e Sudeste: Sapopemba, Itaquera, Lajeado e Itaim Paulista. Os gráficos de óbitos por Covid-19 por distrito estão na Figura 5. A rápida ascensão das curvas dos quatro distritos demonstra que houve uma intensa transmissão inicial da doença, provocando aumento descontrolado de casos na região. São elevados os valores das médias $(16,56 ; 10,62 ; 7,15 ; 9,88$ respectivamente) e dos desvios-padrão (10,74; 7,45; 6,62 e 7,06 respectivamente).

Figura 5 - Curvas de óbitos nos distritos de Sapopemba, Itaquera, Lajeado e Itaim Paulista

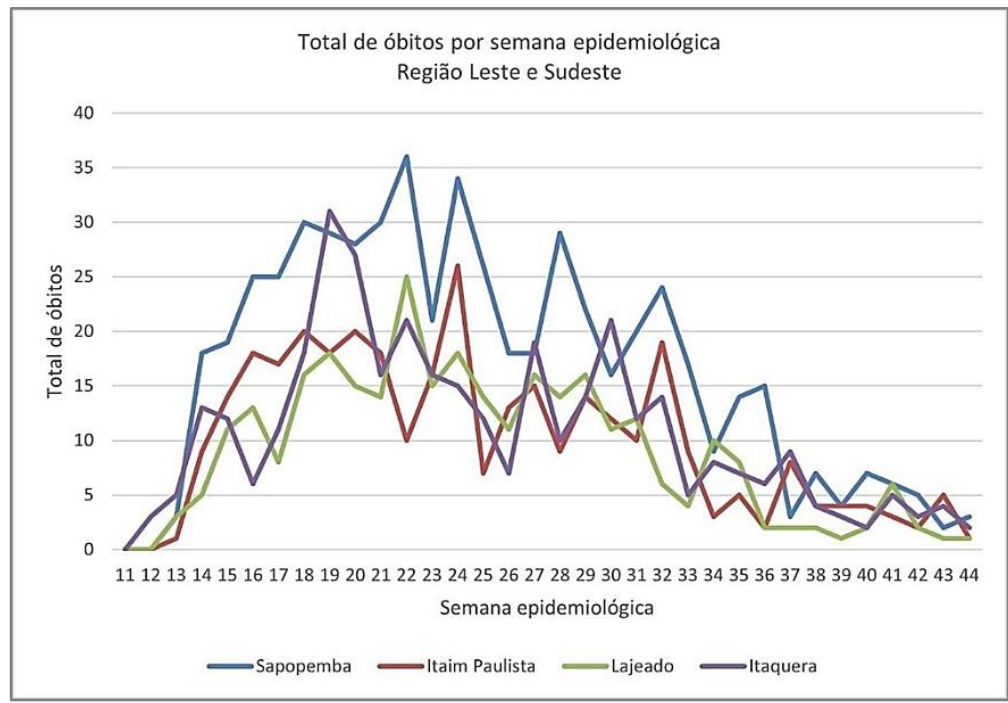

Fonte: Autor a partir de dados do DATASUS (2020) 
Os estudos soroepidemiológicos realizados entre 15 e 24 de junho de 2020 mostraram soroprevalência para Covid-19 de 16\% nos quatro distritos. No estudo repetido entre 01 e 10 de outubro, a soroprevalência passou para 30,4\% nos quatro distritos. O Quadro 5 mostra os dados de epidemiologia, demografia, leitos hospitalares, domicílios, mobilidade e tempo de deslocamento ao trabalho.

Quadro 5 - Dados dos distritos de Sapopemba, Itaquera, Lajeado e Itaim Paulista

\begin{tabular}{|c|c|c|c|c|c|c|c|}
\hline \multirow{2}{*}{ Epidemiologia } & \multirow{2}{*}{$\begin{array}{l}\text { Óbitos até a } 44^{\mathrm{a}} \\
\text { Semana } \\
\text { Epidemiológica }\end{array}$} & \multirow{2}{*}{\multicolumn{3}{|c|}{$\begin{array}{c}\text { Óbitos por } 1.000 \\
\text { habitantes do Distrito }\end{array}$}} & \multicolumn{3}{|c|}{ Óbitos por faixa etária } \\
\hline & & & & & \multicolumn{2}{|c|}{$<65$ anos } & $>65$ anos \\
\hline Sapopemba & 563 & \multicolumn{3}{|c|}{2,0} & 162 & $9 \%)$ & $401(71 \%)$ \\
\hline Itaquera & 361 & \multicolumn{3}{|c|}{1,8} & \multicolumn{2}{|c|}{$133(37 \%)$} & $228(63 \%)$ \\
\hline Lajeado & 302 & \multicolumn{3}{|c|}{1,8} & \multicolumn{2}{|c|}{$128(42 \%)$} & $174(58 \%)$ \\
\hline Itaim Paulista & 336 & \multicolumn{3}{|c|}{1,5} & \multicolumn{2}{|c|}{$129(38 \%)$} & $207(62 \%)$ \\
\hline $\begin{array}{l}\text { Demografia e } \\
\text { hospitais }\end{array}$ & População & \multicolumn{3}{|c|}{ Índice de Envelhecimento } & \multicolumn{2}{|c|}{$\begin{array}{l}\text { Renda } \\
\text { Mensal } \\
\text { Média } \\
\end{array}$} & $\begin{array}{c}\text { Coeficiente } \\
\text { de leitos } \\
\text { gerais } \\
\end{array}$ \\
\hline Sapopemba & 284.524 & \multicolumn{3}{|c|}{69,5} & \multicolumn{2}{|c|}{$1.022,94$} & $\begin{array}{c}0,82 / 1.000 \\
\text { habs }\end{array}$ \\
\hline Itaquera & 204.871 & \multicolumn{3}{|c|}{64,5} & \multicolumn{2}{|c|}{$1.1091,38$} & $\begin{array}{c}1,41 / 1.000 \\
\text { habs }\end{array}$ \\
\hline Lajeado & 164.512 & \multicolumn{3}{|c|}{44,7} & \multicolumn{2}{|c|}{861,38} & $\begin{array}{c}1,06 / 1.000 \\
\text { habs }\end{array}$ \\
\hline Itaim Paulista & 224.074 & \multicolumn{3}{|c|}{51,3} & \multicolumn{2}{|c|}{914,45} & $\begin{array}{c}0,77 / 1.000 \\
\text { habs }\end{array}$ \\
\hline \multirow[t]{2}{*}{ Domicílios } & \multirow{2}{*}{$\begin{array}{c}\text { Domicílios não } \\
\text { ligados à rede } \\
\text { de esgoto }\end{array}$} & \multicolumn{3}{|c|}{$\begin{array}{l}\text { Densidade de pessoas por } \\
\text { dormitório }\end{array}$} & \multirow{2}{*}{\multicolumn{2}{|c|}{$\begin{array}{l}\text { Número de } \\
\text { favelas }\end{array}$}} & \multirow{2}{*}{$\begin{array}{l}\text { Domicílios } \\
\text { em favelas }\end{array}$} \\
\hline & & Até 1 & 1 a 3 & $>3$ & & & \\
\hline Sapopemba & $6.249(7 \%)$ & $24 \%$ & $66 \%$ & $10 \%$ & \multicolumn{2}{|c|}{44} & 18.273 \\
\hline Itaquera & $4.883(8 \%)$ & $25 \%$ & $64 \%$ & $11 \%$ & \multicolumn{2}{|c|}{18} & 4.146 \\
\hline Lajeado & $5.826(12,5 \%)$ & $18 \%$ & $68 \%$ & $14 \%$ & \multicolumn{2}{|c|}{27} & 3.993 \\
\hline Itaim Paulista & $5.529(8,6 \%)$ & $22 \%$ & $67 \%$ & $11 \%$ & \multicolumn{2}{|c|}{21} & 4.808 \\
\hline $\begin{array}{l}\text { Mobilidade e } \\
\text { transporte }\end{array}$ & \multicolumn{5}{|c|}{ Índice de Mobilidade } & \multicolumn{2}{|c|}{$\begin{array}{l}\text { Tempo médio de } \\
\text { deslocamento ao } \\
\text { trabalho }\end{array}$} \\
\hline Sapopemba & \multicolumn{2}{|c|}{$\begin{array}{c}\text { Motorizado Individual } \\
-0,49 \\
\text { Motorizado Coletivo - } \\
0,82\end{array}$} & $\begin{array}{l}\text { A pé } \\
0,61\end{array}$ & $\begin{array}{l}\text { Bicicleta } \\
0,01\end{array}$ & $\begin{array}{l}\text { Total } \\
1,93\end{array}$ & \multicolumn{2}{|c|}{$1: 30$ a $2: 00 \mathrm{~h}$} \\
\hline Itaquera & $\begin{array}{r}\text { Motorizado Indi } \\
-0,34 \\
\text { Motorizado Col } \\
0,78 \\
\end{array}$ & vidual & $\begin{array}{l}\text { A pé } \\
0,62\end{array}$ & $\begin{array}{c}\text { Bicicleta } \\
0\end{array}$ & $\begin{array}{c}\text { Total } \\
1,74\end{array}$ & & a 2:00 h \\
\hline
\end{tabular}




\begin{tabular}{|c|c|c|c|c|c|}
\hline \multirow{2}{*}{ Lajeado } & $\begin{array}{c}\text { Motorizado Individual } \\
-0,34\end{array}$ & $\begin{array}{c}\text { A pé } \\
0,71\end{array}$ & $\begin{array}{c}\text { Bicicleta } \\
0,02\end{array}$ & $\begin{array}{c}\text { Total } \\
1,83\end{array}$ & $2: 00$ a $2: 30 \mathrm{~h}$ \\
\hline \multirow{3}{*}{ Itaim Paulista } & 0,76 & & \\
& $\begin{array}{c}\text { Motorizado Individual } \\
-0,38\end{array}$ & $\begin{array}{c}\text { A pé } \\
0,70\end{array}$ & $\begin{array}{c}\text { Bicicleta } \\
0\end{array}$ & $\begin{array}{c}\text { Total } \\
1,90\end{array}$ & $2: 00$ a $2: 30 \mathrm{~h}$ \\
& 0,81 & & & \\
\hline
\end{tabular}

O conjunto de dados mostra que as curvas de óbitos caracterizadas por início acelerado e picos proeminentes se associaram a más condições socioeconômicas da população, elevada porcentagem de domicílios não ligados à rede de esgoto, alto número de favelas, alta densidade de pessoas por dormitório e uso do transporte coletivo motorizado como principal meio de locomoção para o trabalho, associando-se a tempo de deslocamento elevado. O baixo coeficiente de leitos hospitalares em todos os distritos, também pode ter contribuído para esse comportamento da curva de óbitos e para a elevada mortalidade, apresar do baixo Índice de Envelhecimento da população.

\section{DISCUSSÃO DOS RESULTADOS E CONCLUSÃO}

A epidemia de Covid-19 afetou de forma diferente os vários distritos do Município de São Paulo. O ritmo de disseminação da doença e a curva de óbitos não dependeram apenas da presença do coronavírus, mas foram decorrentes das condições existentes em cada distrito.

Nas regiões urbanas mais periféricas, principalmente na zona Leste e nos extremos das zonas Norte e Sul, observou-se disseminação mais rápida da Covid-19, produzindo curvas de óbitos caracterizadas por rápida aceleração inicial, progredindo para picos acentuados. Por outro lado, nos distritos mais consolidados das regiões Centro-oeste e Sudoeste, as curvas de óbitos tiveram padrão achatado, demostrando os efeitos da implementação das medidas de distanciamento social, com redução na transmissão do vírus.

Diversos fatores mostraram relação com o maior impacto da epidemia de Covid19 nas regiões periféricas do MSP, em especial o baixo nível socioeconômico, a precariedade das habitações, a alta densidade de pessoas por domicílio. o compartilhamento do dormitório por muitas pessoas, a falta de infraestrutura urbana, o tempo e a forma de locomoção para o trabalho, os problemas de mobilidade urbana e a carência da rede de atendimento hospitalar. 
Entre os fatores demográficos associados à maior mortalidade por Covid-19, destaca-se o maior índice de envelhecimento da população. Este fato se deve à maior ocorrência de comorbidades e de complicações evolutivas da doença na população idosa. Nos distritos com maior índice de envelhecimento, mesmo na presença de altos coeficientes de leitos hospitalares, excelentes condições de moradia e opções de transporte individual, não foi possível reduzir a mortalidade nesta faixa etária. Nos distritos de Jardim Paulista, Moema e Pinheiros, 90\% dos mortos tinham mais de 65 anos.

Os estudos soroepidemiológicos mostraram maior soroprevalência nas regiões mais vulneráveis, comprovando a maior dificuldade de implementação das medidas de controle da disseminação da doença. Essas medidas se tornam impraticáveis nas comunidades em que ocorrem problemas na qualidade das habitações, baixos níveis socioeconômicos, falta de de infraestrutura e indisponibilidade de meios de transporte individuais. $\mathrm{O}$ coeficiente de leitos hospitalares também é um fator importante para o tratamento eficiente dos doentes, evitando as complicações evolutivas que se associam com maior mortalidade.

Diversos temas precisam ser discutidos no planejamento das cidades, incluindo a extensão dos serviços públicos essenciais a todas as áreas habitadas do município, a flexibilidade de uso dos espaços públicos, o melhor dimensionamento das áreas destinadas a pedestres e ciclistas, as políticas urbanas voltadas para melhoria das condições de moradia da população de baixa renda e a melhor distribuição da rede pública de assistência médica e de leitos hospitalares.

Pensando na possível ocorrência de futuras epidemias, é essencial que se adotem medidas para a correção das condições responsáveis pela maior vulnerabilidade de algumas parcelas da população. O estímulo para a transformação dos bairros dormitórios de baixa renda em regiões multifuncionais também é importante. Essa medida, além de gerar empregos e renda, também ajuda a evitar o longo tempo de deslocamento até o trabalho.

\section{REFERÊNCIAS}

ALIROL, E., et al. Urbanisation and infectious diseases in a globalised world. The Lancet Infectious Diseases, v. 11, n. 2, p 131-141, 2011.

BONACCORSI, G., et al. Economic and social consequences of human mobility restrictions under COVID-19. Proceedings of the National Academy of Sciences, v.117, n. 27, p. 15530-15535, 2020. 
FERGUSON N. M., et al. Impact of non-pharmaceutical interventions (NPIs) to reduce COVID- 19 mortality and healthcare demand. Imperial College COVID-19 Response Team, v. 16, 2020.

GONÇALVES, E. L., et al. assistência hospitalar no âmbito da previdência social no Estado de São Paulo, Brasil. Rev. Saúde Pública., v. 6, p. 35-43, 1972.

JARDIM, V. C., et al Análise sistêmica do município de São Paulo e suas implicações para o avanço dos casos de Covid-19. Estudos Avançados, v. 34, n. 99. 2020.

JIANG, J.; LUO, L. Influence of population mobility on the novel coronavirus disease (COVID-19) epidemic: based on panel data from Hubei, China. Global Health Research and Policy, v. 5, n.1, 2020.

KRAEMER, M. U. G., et al. The effect of human mobility and control measures on the COVID-19 epidemic in China. Science, v. 368, p. 493-497, 2020.

NUSSBAUMER-STREIT, B., et al. Quarantine alone or in combination with other public health measures to control COVID-19: a rapid review. Cochrane Database Syst Rev., v. 8; n. 4, 2020.

PAN, A., et al. Association of Public Health Interventions with the Epidemiology of the COVID-19 Outbreak in Wuhan, China. JAMA: 323, n. 19, p. 1915-1923, 2020.

VERITY, R., et al. Estimates of the severity of COVID-19 disease. The Lancet, v. 20, n. 6, p. 669-677, 2020. COMPANHIA AMBIENTAL DO ESTADO DE SÃO PAULO - 